The submitted manuscript has been authored by a contractor of the U.S. Government under contract No. W-31-109ENG-38. Accordingly, the U.S. Government retains a nonexclusive, royalty-free license to publish

\title{
Technical Specification for Plate Fabrication For the Atlas Tile Hadron Calorimeter
}

13 June 1995

Prepared by: Norman F. Hill

High Energy Physics Division Argonne National Laboratory 9700 South Cass Avenue Argonne Illinois, 60439 USA

* Work supported by the U.S. Department of Energy, Division of High Energy Physics, Contract W-31-109-ENG-38. 


\section{DISCLAIMER}

This report was prepared as an account of work sponsored by an agency of the United States Government. Neither the United States Government nor any agency thereof, nor any of their employees, make any warranty, express or implied, or assumes any legal liability or responsibility for the accuracy, completeness, or usefulness of any information, apparatus, product, or process disclosed, or represents that its use would not infringe privately owned rights. Reference herein to any specific commercial product, process, or service by trade name, trademark, manufacturer, or otherwise does not necessarily constitute or imply its endorsement, recommendation, or favoring by the United States Government or any agency thereof. The views and opinions of authors expressed herein do not necessarily state or reflect those of the United States Government or any agency thereof. 


\section{DISCLAIMER}

Portions of this document may be illegible in electronic image products. Images are produced from the best available original document. 


\section{Project Description}

The Atlas Collaboration, at the European Organization for Nuclear Research (CERN), proposes to build a general purpose proton-proton detector for the Large Hadron Collider (LHC), located in Geneva, Switzerland. The ATLAS collaboration consists of approximately 100 international institutions (universities and research laboratories) with a worldwide distribution. The ATLAS detector includes a large scintillating tile calorimeter using iron as the absorber material. This detector will be used to identify and measure the by-products of proton-proton collisions that occur at the symmetric center. The design of this detector is unique in that the absorber plates are oriented perpendicular to the colliding beam axis, rather than parallel, as is done in most other similar detectors to date. A simplified view of the detector is shown in the figure below.

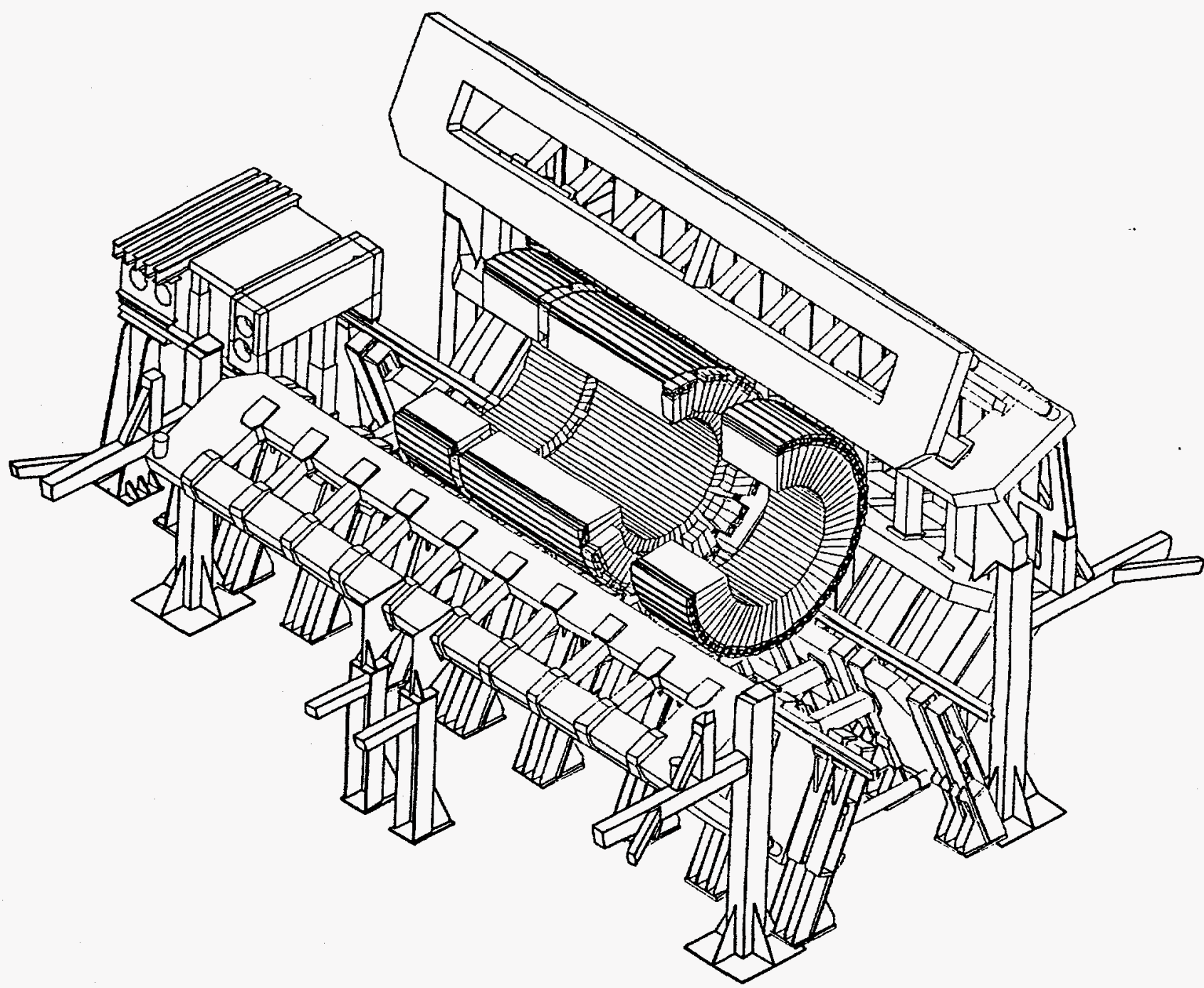

Figure 1 


\section{Tile Calorimeter Description}

The Tile-Calorimeter consists of a cylinder constructed of steel plates that are trapezoidal in shape and formed into wedge shaped structures that are $2283 \mathrm{~mm}$ at their inner radius and $4230 \mathrm{~mm}$ at their outer radius. Each wedge module in the barrel is constructed of 19 submodules approximately $30 \mathrm{~cm}$ thick. The extended barrel portion of the detector consists of nine submodules, approximately $30 \mathrm{~cm}$ long, that form a similar cylinder that extends from each end of the barrel. The barrel cylinder is $5639.2 \mathrm{~mm}$ long, and each of the extended barrel cylinders is $2650 \mathrm{~mm}$ each. Each cell in the construction consists of two long trapezoidal plates $5 \mathrm{~mm}$ thick. A series of shorter plates $4.05 \mathrm{~mm}$ thick are staggered in between to form a checkerboard pattern when assembled. Each submodule is stacked with a specified number of cells or periods arrayed along the barrel axis. The total weight of the steel plates in the detector is approximately 3000 metric tons.

\section{Organization}

The U.S. participation in the Atlas Collaboration will require that fabrication of the plates into the appropriate shapes, plus the assembly into the prescribed modules be carried out by institutions in the U.S. At the present time the U.S. institutions responsible for this effort are: Argonne National Laboratory, the University of Chicago, the University of Illinois, Michigan State University, and the University of Texas, Arlington.

\section{Technical Specification}

The punched steel plates that will be used to construct the calorimeter described above, have applied tolerancing that is more stringent than is normally encountered for these manufacturing processes. It is therefore incumbent on the fabricator to understand the tolerancing and its effect on the overall assembly. The tolerancing for the location of the holes, keyways, and overall plate dimensions are identified on the applicable drawings.

\section{Applicable Drawings}

Drawing

Description

AT-10-1-12 (Attachment 1)

Atlas Module Assembly Plate Master 


\title{
Material
}

The material to be used for fabricating these plates is identified in the "Technical Specifications for the Supply of Steel for the ATLAS Tile Hadron Calorimeter" (Attachment \# 2). This material will be furnished by Argonne National Laboratory and will be supplied by a mill, or mills, in Europe. The general description of the plates is that of hot rolled, low carbon steel plate. Additional cold reduction will most likely be used to achieve the required thickness tolerance. The material will be furnished in either coil or sheet form, although sheet is preferred. If the successful vendor has flattening and shearing capability this price should be itemized separately.

\section{Quantity Required}

\author{
Master Plates \\ 1200 pcs.
}

Drawing \# AT-10-1-12

This quantity represents only a prototype amount, and the total production quantity can be as many as 78,000 plates and as few as 38,000 . In order to estimate the eventual cost of these plates, each vendor is also asked to quote a price for production runs of 78,000 and 38,000 pieces.

\section{Plate Punching}

The master plates (Drawing \# AT-10-1-12) are to be die punched in two steps. The first step will produce the holes and keyways at both the small and large end of the plate, and the second step will trim the plate size to final dimension. This second step is necessary, since the key slots punched in the first step will be used to maintain the required tolerances on the width, included angle, and symmetry about the centerline of the finished plate. If the successful vendor can demonstrate that the dimensions and tolerances can be satisfied with a single die, he should so state.

\section{Flattening, Deburring, and Finishing}

All out of plane distortions, including burrs, must be removed from the plates after punching. The use of abrasive belt grinding is suggested for this process for example, equipment manufactured in the U.S. under the trade name of "Time 
Saver". Upon completion of this step, no out of plane distortions should be detectable when a straight edge is passed over the plate in both the radial $(Y)$ and the $\phi(X)$ directions. The Time Saver process may also be used to provide the necessary finish for final bonding of these plates into the $30 \mathrm{~cm}$ stacks. Since the deburring operation is required, the vendor may also be asked to provide this finish as final preparation of the plates before shipping.

\section{Stacking and Sorting}

Since the steel may be received in either coil or sheet, and in order to eliminate the necessity for sorting and restacking the punched plates after punching, it is preferred that systematic anomalies (taper for example) be eliminated at the time of flattening and shearing the plates from the coil. If this flattening and shearing is done by the vendor before punching them, systematics should be eliminated during that operation by flipping every other sheet. This orientation should then be maintained after punching. If the steel is received in precut sheets, the orientation as received should be maintained.

\section{Vendor Responsibility}

It will be the responsibility of the successful vendor to fulfill all of the requirements of this specification, if subcontractors are used for any part of required fabrication, they must be identified as part of the bid process.

\section{Proving the Die}

The die accuracy, and compliance to the drawing dimensions and tolerances, will be proven by a punching of at least ten plates prior to starting the production run. This proving of the die will be witnessed by an Argonne Representative upon notification by the vendor.

\section{Acceptance}

Acceptance of the finished plates will be based on a random final inspection of at least $10 \%$ of the plates. This inspection can be based on a witnessed inspection at the vendors facility before shipment by Argonne personnel, or by certification of that $10 \%$ inspection by the vendor. The top ten plates of every pallet should be 
reserved for the inspected plates (see packaging for shipment). Acceptance of any plates that do not meet the specified dimensional tolerances will be at the discretion of Argonne National Laboratory.

\section{Protection}

After finishing, the plates must be protected from corrosion. It is therefore required that they be sprayed with oil and stacked with oiled paper between each plate before packaging for shipment. The oil to be used will be specified by Argonne as it is dependent on removal technique, however normal oils used in the stamping process will most likely be acceptable as long as they do not have substantial paraffin content.

\section{Packaging for Shipment}

The finished plates will be stacked on pallets that are of sufficient dimension to contain the plates with no overhang that could result in damage. Since some portion of these plates will be shipped to locations outside the U.S., each pallet should have a box cover that can be banded to the supporting pallet. The suggested load for each pallet is 100 plates, or no more than $4500 \mathrm{lbs}$. or $2050 \mathrm{kgs}$. The ten (10) inspected plates will be stacked on top of each pallet prior to packaging for shipment.

Delivery of all 1200 plates is contingent upon an expected delivery of the steel by the end of September, 1995. The required delivery for all 1200 stampings is no later than 31 October 1995. Partial deliveries will be accepted prior to that date.

\section{Shipping}

All plates will be shipped to Argonne National Laboratory at the following address:

Argonne National Laboratory

9700 South Cass Avenue

Argonne, Illinois 60439

Attention: Norman F. Hill 362/HEP 


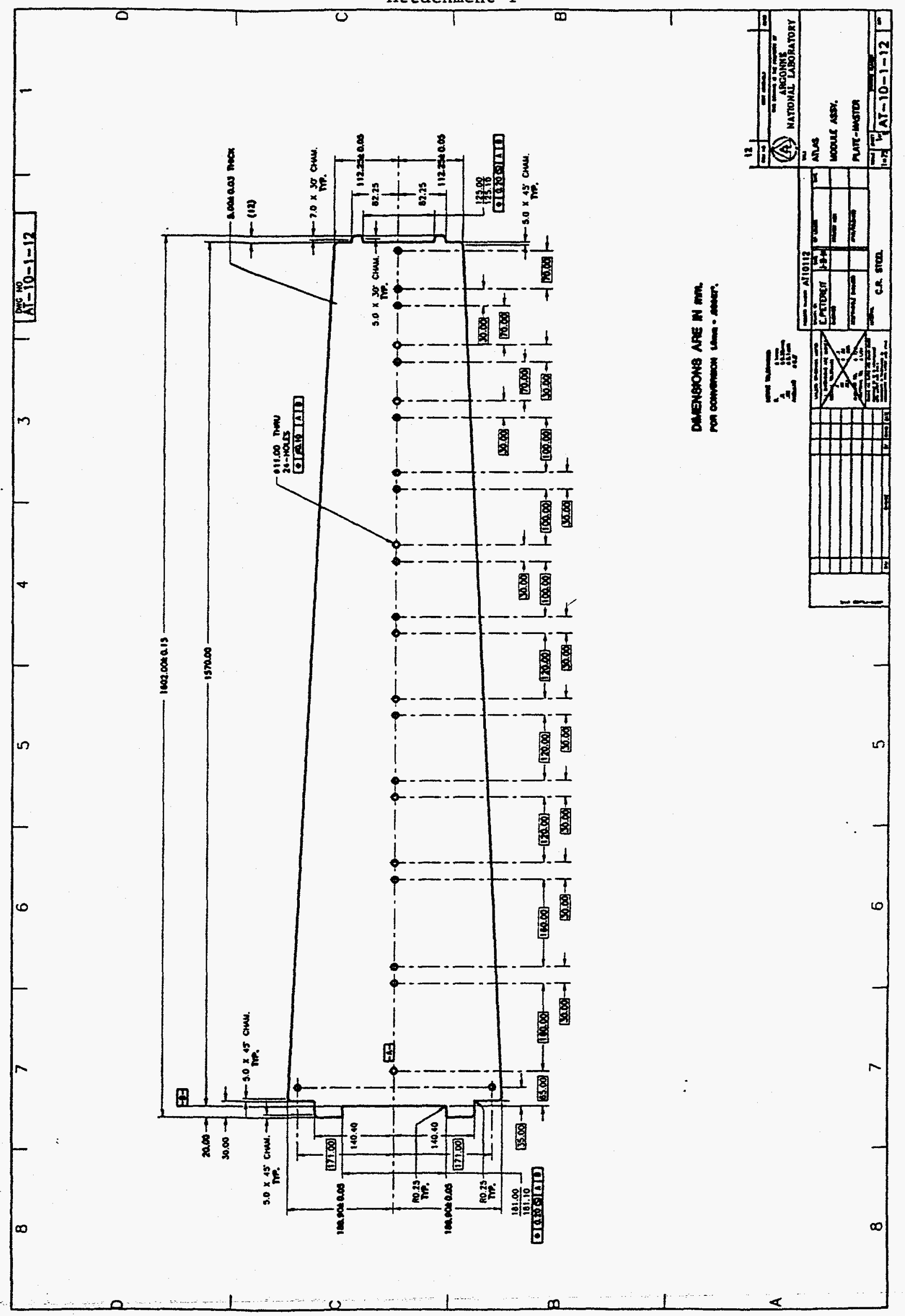




\title{
EUROPEAN ORGANIZATION FOR NUCLEAR RESEARCH
}

\section{ATLAS Internal Note TILECAL-NO-xxx 06-April-95}

\author{
Technical Specifications \\ for the supply of steel for \\ the ATLAS tile hadron calorimeter
}

Atlas, Tilecal collaboration 


\section{INTRODUCTION AND SCOPE OF THE STUDY}

\subsection{The ATLAS project}

The ATLAS collaboration at CERN proposes to build a general purpose proton-proton detector for the Large Hadron Collider (LHC), capable of exploring a new energy region which will become accessible. ATLAS is an international collaboration consisting of about 150 institutions (University groups and Research laboratories) distributed through out much of the world. The ATLAS detector facility, one of two detectors, includes a large, plastic scintillating tile hadron calorimeter using steel as showering material. This detector will perform the task of measuring jets and missing transverse energy. The chosen technology is based on a sampling technique, using wavelength shifting fibers to readout the plastic scintillating tiles. The unique characteristic of this design is the unusual orientation of the scintillator tiles. The usual orientation is parallel to the detector axis whereas in this detector the plates are perpendicular.

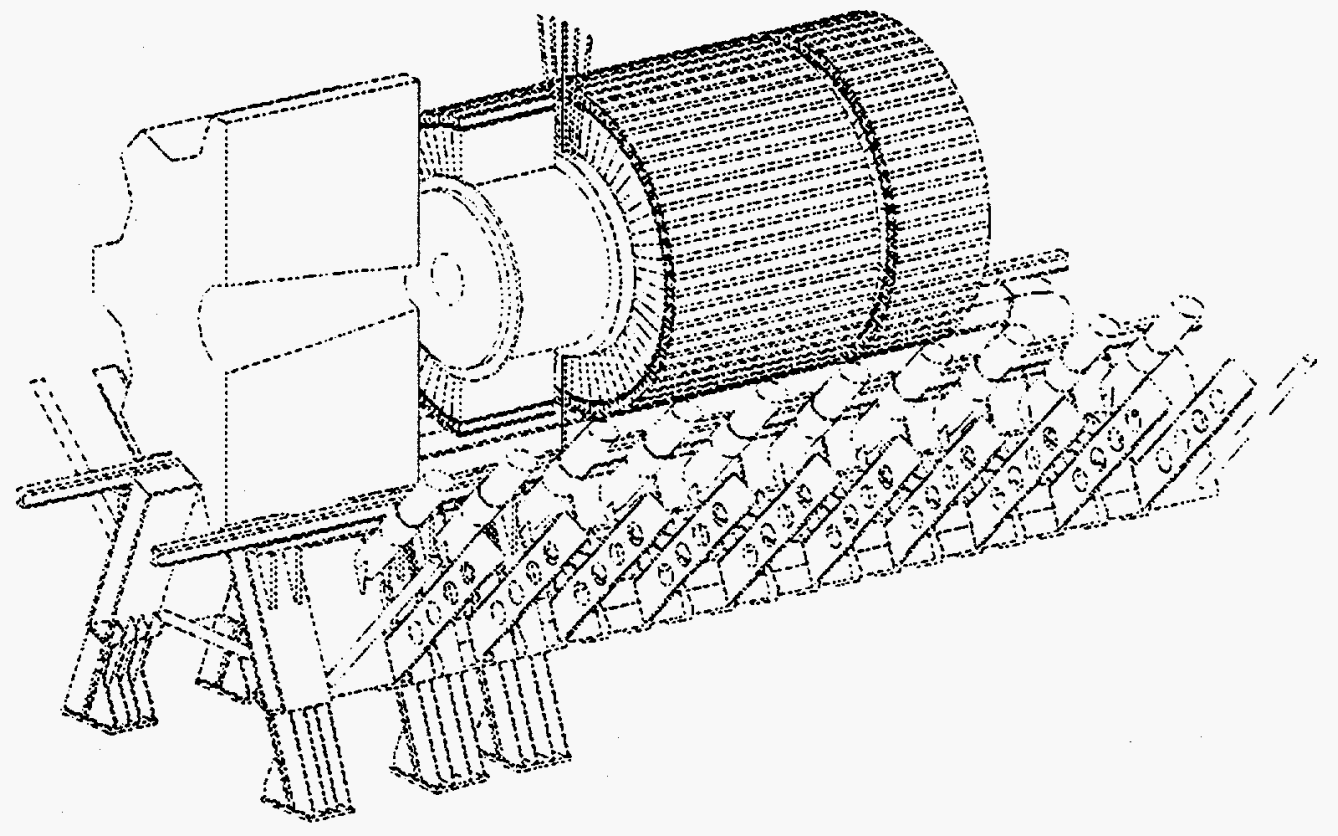

\subsection{Scope of these technical specifications}

This document summarizes the requirements and specifications for the steel needed to construct the TILE calorimeter for the detector. Approximately 2800 metric tonnes of common low carbon steel must be supplied in the form of sheets or coils, ready for further processing, by either stamping or laser cutting into the final configuration. For mechanical reasons in the later fabrication and assembly, of the detector, low internal stresses in the steel are important. The flatness and uniformity of thickness are also important requirements for maintaining the dimensions of the final laminated stacking. The surface of the steel must be free of rust and protected against corrosion in anticipation of intermediate storage. 


\section{GENERAL DESCRIPTION}

\subsection{The ATLAS tile hadron calorimeter}

Mechanically the calorimeter consists of a cylindrical structure with an inner radius of $2280 \mathrm{~mm}$ and an outer radius of $4230 \mathrm{~mm}$. It is divided into one (1) $5640 \mathrm{~mm}$ long barrel part with two (2) $2650 \mathrm{~mm}$ long extended barrel parts, one at each end. Each of these units is further subdivided into sixty four (64) independent sector modules. The steel structure of the active part of each six (6) meter sector module (for example the barrel module) consists of an assembly of nineteen (19) independent submodules approximately $300 \mathrm{~mm}$ in thickness which is constructed using 16 basic "periods". A period is described as an assembly of four (4)laminated steel layers The first and third layers being formed by large trapezoidal steel plates, $5 \mathrm{~mm}$ thick, spanning the full radial dimension of the module $(1600 \mathrm{~mm})$ and referred to here as master plates. The second and fourth layer consist of smaller trapezoidal steel plates and scintillator tiles alternating along the radial dimension. The smaller steel trapezoids (spacer plates) are $4.05 \mathrm{~mm}$ thick with 13 different sizes. The completed structure is achieved by glueing spacers to masters plates in an alternating pattern. Four straps welded to each of the 4 corners of the submodules complete their fabrication.

\subsection{Materials, manufacturing and tolerances}

- base material : Ordinary low-carbon rolled steel ( $\mathrm{Fe} 360$ or equivalent, defined by the Euronorm EN10025). The chemical composition is defined in this norm, the main aspects are (the values are the maximum allowed):

$C=0.20 \% \quad P=0.05 \% \quad S=0.05 \% \quad N=0.009 \%$

The mechanical characteristics are defined by this norm as:

$R e=235 \mathrm{Mpa} \quad R m=340 \mathrm{Mpa}$

All steel supplied for this specification must have the same chemical composition and must have undergone the same heat treatment. The normal magnetic properties of this type of steel are sufficient.

- production conditions : hot or cold rolled steel, delivered in sheets or coils . Part of this steel will be $5.00 \mathrm{~mm}$ thick, part $4.05 \mathrm{~mm}$ thick. Each sheet will be associated to a production batch, normally a coil. The definition of a production batch will be agreeded upon with the producers.

- quantity

- 78000 steel sheets of $1620 \mathrm{~mm} \times 400 \mathrm{~mm} \times 5.00 \mathrm{~mm}$ for a total of about 1989 tons.

- 39500 steel sheets of $1620 \mathrm{~mm} \times 400 \mathrm{~mm} \times 4.05 \mathrm{~mm}$ for a total of about 816 tons. 


\section{- quality and tolerances}

Particular tolerance requirements are associated with this procurment. Two approaches to the problem are proposed:

- The average thickness of a single batch will deviate by no more than \pm 0.010 $\mathrm{mm}$, from the nominal thickness of $4.05 \mathrm{~mm}$ and $5.00 \mathrm{~mm}$. The definition of this requirement is part of the inspection section (Global tolerance), this will guarantee the uniformity of production.

- The range of thickness within a single sheet (Local tolerance) must be kept within $\pm 0.030 \mathrm{~mm}$ of the nominal value.

Inspection of a subsample of sheets for each batch will be required to ensure adherence to the required quality. In the case of steel furnished in coils coupons cut from these coils will be required to perform similar inspections. The requirements for quality assurance are defined differently for sheets and coils. Those differences are explained as follows.

1. For sheets it will be required that the first middle and last sheet cut from the original coil will be measured for thickness and flatness. The information recorded from these measurements along with the continuous thickness monitoring during rolling will provide both a check against calibration and a history of the coil.

2. For coils wound after rolling, it will be necessary to cut a plate from both the beginning and end of each coil. These plates will then be measured and the information used in the same way as described above.

requirments on good surface quality and flatness, as well on low internal stresses are defined in the quality checks section below.

- shipping and storage: short-term storage must be provided to allow shipping to the final cutting and assembly plants located in the USA, Russia and central Europe. As part of the quoted price the producer will be responsible for the packing and preparation of the steel for transportation. The preparation for shipment will include the oiling of the plates or coils prior to packing and shipment. It should be pointed out that the shipments of these plates or coils will subject a variety of environments

Surface protection from oxidation is also a part of the procurment and must be agreed upon with ATLAS; however the primary way chosen to protect the steel is with oil as described above.

- supplementary information: associated to each material lot the manufacturer must provide the following information:

- Heat number to provide a history of production

- Chemical composition certificate 
- Material history, including thermal and mechanical treatments and storage conditions.

- Online thickness measurment protocols and copies of the online data.

- Quality reports

\section{QUALITY CHECKS and REQUIREMENTS}

Several quality checks (QC) on thickness and fiatness tolerances as well as internal stresses and surface quality must be performed by the manufacturer on each batch. The manufacturer shall be entirely responsible for the correct manufacturing of the steel sheets as described in this specification. ATLAS will reserve the right to reject batches or individual sheets not corresponding to the specifications. Typically for each batch the first, the middle and the last steel sheet as described earlier in this specification, will be used for this quality inspection. In the case of rerolled coils a sheet will be cut from the beginning and end of each coil after waste has been trimmed.

- Global tolerances : The manufacturer will provide for each batch an online set of measurments made during production, on paper or on computer medium. This measurement which is normally done during production must demonstrate that the entire length of the roll, and the average longitudinal profile as measured on a line along the rolling direction is kept within $\pm 0.010 \mathrm{~mm}$ from the nominal value. All systematic drifts from the nominal setting will also be kept within $\pm 0.010 \mathrm{~mm}$.

- Local tolerances : the thickness of the 3 sheets per batch should be measured on 12 points as indicated in Figure 1. The distance from the sheet edges is 20 $\mathrm{mm}$. This measurements can be made using a micrometer or an ultrasonic system. From these 12 measurements, 2 values should be retained:

- average thickness tolerance $:\left|T K_{\text {nominal }}-T K_{\text {average }}\right| \leq 0.010 \mathrm{~mm}$.

- thickness dispersion: $Q 1=\left(T K_{\max }-T K_{\text {nominal }}\right)$, where max is the maximal deviation from the nominal value measured on the 12 points. $|Q 1|$ will not exced $0.030 \mathrm{~mm}$ for the measured sheets.

- internal stresses : these will be measured by cutting two of the steel sheets along both axes of symmetry in 2 parts (different axes for different sheets) The displacements of the 2 halfs which are then put in contact should not exceed 0.3 $\mathrm{mm}$ (see figure 4). The sheets shall be as free as possible from internal stresses in order to avoid deformations of the edges of the sheet after final precision cutting.

The frequency of these measurements must be in principle associated to each batch. During production, in agreement with ATLAS, this measurment can be done every few batches, if results demonstrate compliance with the specification.

- surface flatness : the sample sheet, laid flat on a marble plane without forces applied, will have to present a perfectly developable surface. Furthermore, the 
amplitude of any local ondulation cannot exceed $0.5 \%$ of the local wavelength. The wavelength should not be less than $800 \mathrm{~mm}$ in the case of an undulation in the middle of the sheet, and not less than $400 \mathrm{~mm}$ in the case of undulations on the edge of the sheet (see figure 2). In any case the amplitude can not exceed 5 $\mathrm{mm}$.

- surface quality : The surfaces of all sheets must be free of visible defects and rust, and must be protected against corrosion. The vendor is invited to propose a suitable process, but the primary process will be oil. Each sheet must have clean surfaces, without irregularities and bumps, in order to assure good stacking. The surface roughness must be of N10 quality according to norm UNE 1037 (E) (which means $R_{a}=12.5 \mu$, see figure 3 for definitions).

\section{ACCESS TO INFORMATION and RESPONSI- BILITIES}

For the items covered by these specifications, ATLAS must be informed about all technical details and any subcontracts.

During the manufacturing phase, periodical reports shall be sent to ATLAS on the progress according to a predetermined schedule. ATLAS also requests the right of access to the vendors facility during manufacturing.

The manufacturer shall be entirely responsible for the correct manufacture of the items described in these specifications. ATLAS reserves the right to reject all sheets which are part of a batch wherein the quality checks on the sample sheets as described above are not in compliance with the specification.

In order to prove the validity of the manufacturing procedure, some prototype steel sheets will be required.

Before beginning production the manufacturer will required to provide ATLAS with all detailed work plans and schedules.

The prospective vendors are invited to supply information on their suggested manufacturing procedure as well as any comments on these specifications or suggested modifications. 


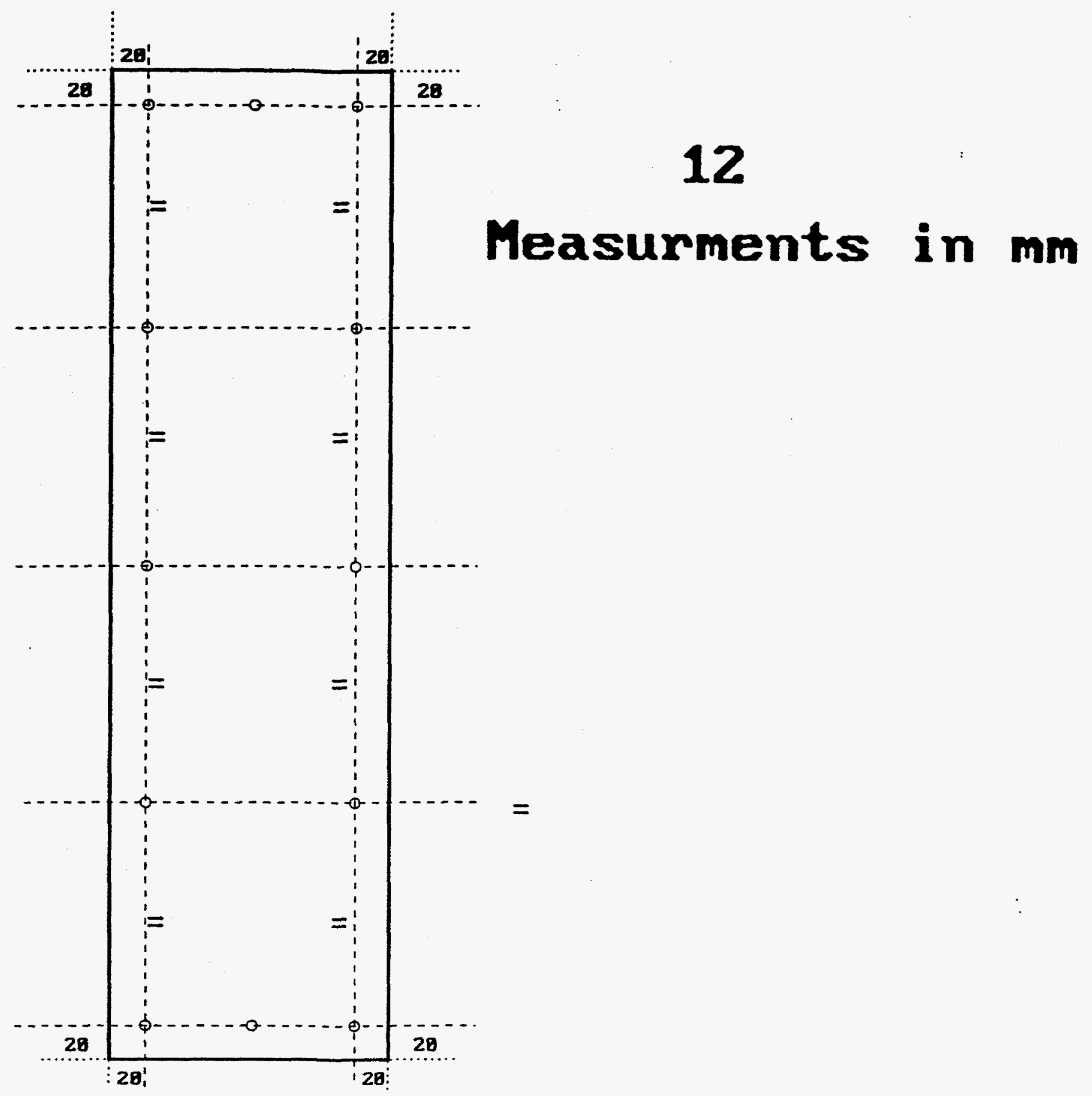

Figure 1: Control poits for thickness. 


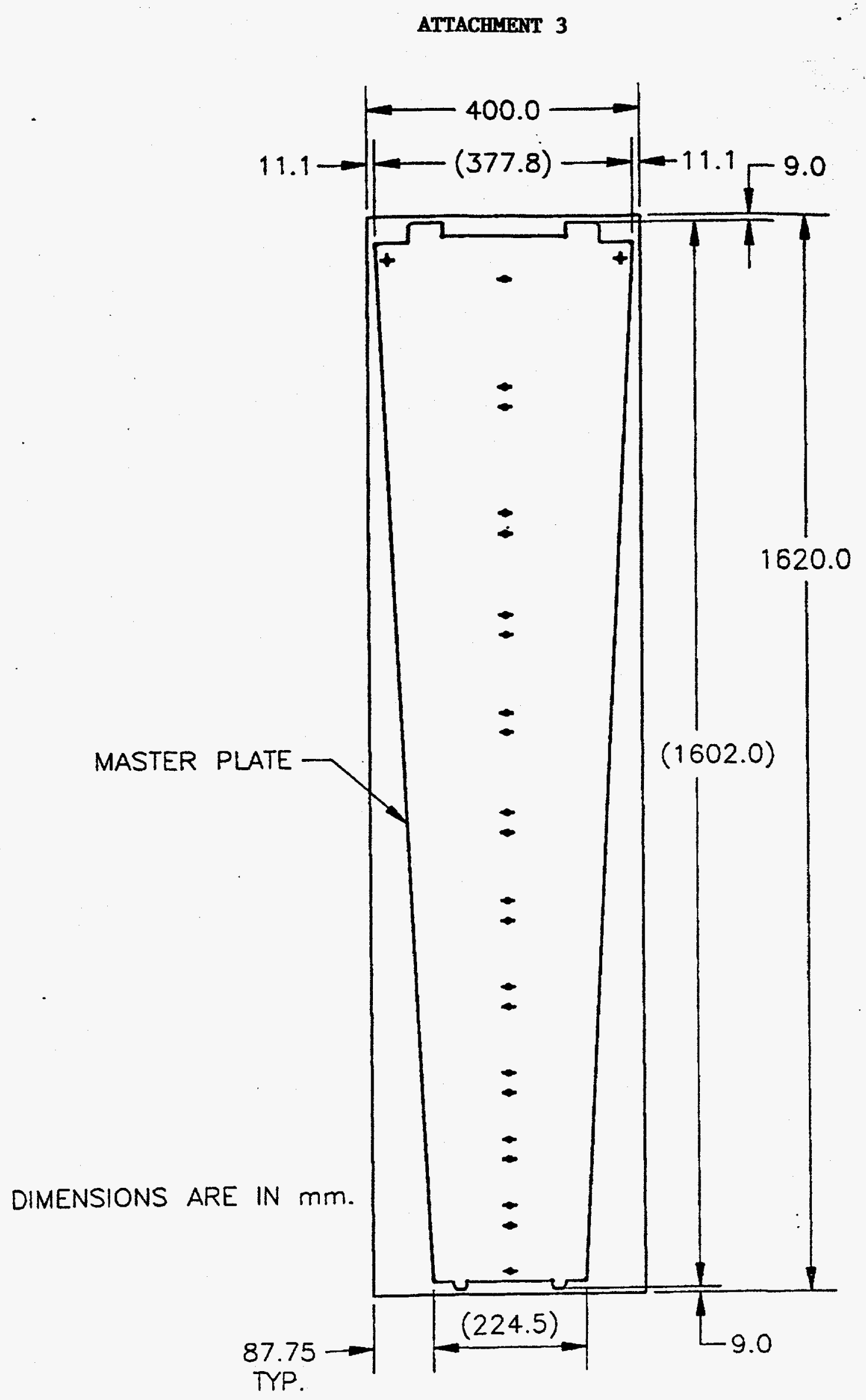

